\title{
Analysis on the Influence of the Height of Tower on Passive Interference in shortwave
}

\author{
Lu Ying ${ }^{1}$, Zhao Zhibin ${ }^{1}$, Zhang Jian gong ${ }^{2}$ and Gan Zheyuan ${ }^{2}$ \\ ${ }^{1}$ College of Electrical and Electronical, North China Electric Power University, Beijing 102206, China \\ ${ }^{2}$ China Electric Power Research Institute, Wuhan, China
}

\begin{abstract}
The passive interference of transmission lines to nearby radio stations may affect the effective reception and transmission of radio station signals. Therefore, the accurate calculation of the electromagnetic scattering of transmission lines under the condition of external electromagnetic waves is the basis for determining the reasonable avoidance spacing of the two. For passive stations operating in shortwave frequencies, passive interference is mainly generated by the tower. This paper uses the method of moments to perform passive interference calculations under normal circumstances, And elaborates the method for calculating the electromagnetic field of the transmission line, obtains the space electric field intensity of the transmission line at the same working frequency and space location of the plane wave. Uses the approximate formula to inductive the formula for calculating height of tower and the protective distance.
\end{abstract}

\section{Introduction}

In recent years, with the rapid development of science and technology, massive construction of infrastructure such as transmission lines and radio stations has led to a close distance between the two[1].In this case, even if the transmission line is not energized, its huge metal structure will affect the reception or transmission of signals from nearby radio stations. This effect caused only by the scattering of the metal conductors of the transmission line is called passive interference[3]. How to determine the change of the avoidance distance between the two due to the different transmission span is the key to the consideration of power line construction.

In response to this problem, the United States[46],Canada[7],Japan[8,9] and other countries have carried out related research on the influence of power lines on signals in different frequency bands such as MF, VHF/UHV, and $\mathrm{m}$ waves since 1960s. The research object involves radio broadcasting[7], radar navigation[6] and other fields[8]. Since the construction of UHV transmission lines in China, China Electric Power Research Institute Wuhan Branch, North China Electric Power University and China Three Gorges University have conducted a systematic and in-depth research on the electric field strength characteristics of UHV transmission lines, and proposed the avoidance standard for $1000 \mathrm{kV}$ AC transmission lines.

In recent years, with the gradual improvement of the voltage level of transmission lines in China and the continuous construction of UHV AC and DC transmission lines, the impact of UHV transmission lines on passive interference of radio stations has become increasingly serious[10-11]. At present, the electromagnetic radiation problem of transmission lines to radio facilities has become one of the most concerned priorities of various departments. The passive interference generated by the transmission line is mainly divided into two aspects: electromagnetic scattering generated by the iron tower and electromagnetic scattering generated by the overhead conductor. According to the correlation and correlation analysis of the electric field strength curves of the short-wavelength transmission lines based on various single macroscopic structures, it can effectively explain that the influence of the range on the passive interference resonance phenomenon in the short-wave band is most significant, that is, from the perspective of macrostructure. the tower is the decisive influence factor[8] of the passive interference of the transmission line in the short-wave band. Therefore, in the short-wave frequency range, the study of the influence of the height of the tower on passive interference is the key to study the electromagnetic radiation generated by the transmission line in the short-wave frequency.

\section{Modelling}

\subsection{Principle analysis}

The passive interference generated by the transmission line is mainly caused by the electromagnetic radiation of the iron tower, which is related to the frequency, polarization mode and relative position of the affected signal. Therefore, the frequency of the electromagnetic 
wave is set to a certain frequency point, and the polarization mode is vertical polarization. According to the correlation degree and correlation analysis of passive interference curves of the short-wavelength transmission lines based on various single macroscopic structures, studying on the passive interference of tower height to radio stations by changing the height of the transmission line. The effects of electromagnetic interference are: $S=20 \log \frac{E_{Y}}{E_{\mathrm{N}}}$

$E_{Y}$ indicates that the transmission line and the impact of the tower affect the spatial electric field strength of the observation point; $E_{N}$ indicates that the transmission line and the tower affect the spatial electric field strength of the previous observation point.

\subsection{Tower type selection and parameters}

Since there is no relevant standard reference, the typical tower type of the horn tower in the AC transmission line is used in calculation. The height of the tower is $98.5 \mathrm{~m}$, the structure and parameters of the AC transmission line tower are shown in Figure 1.

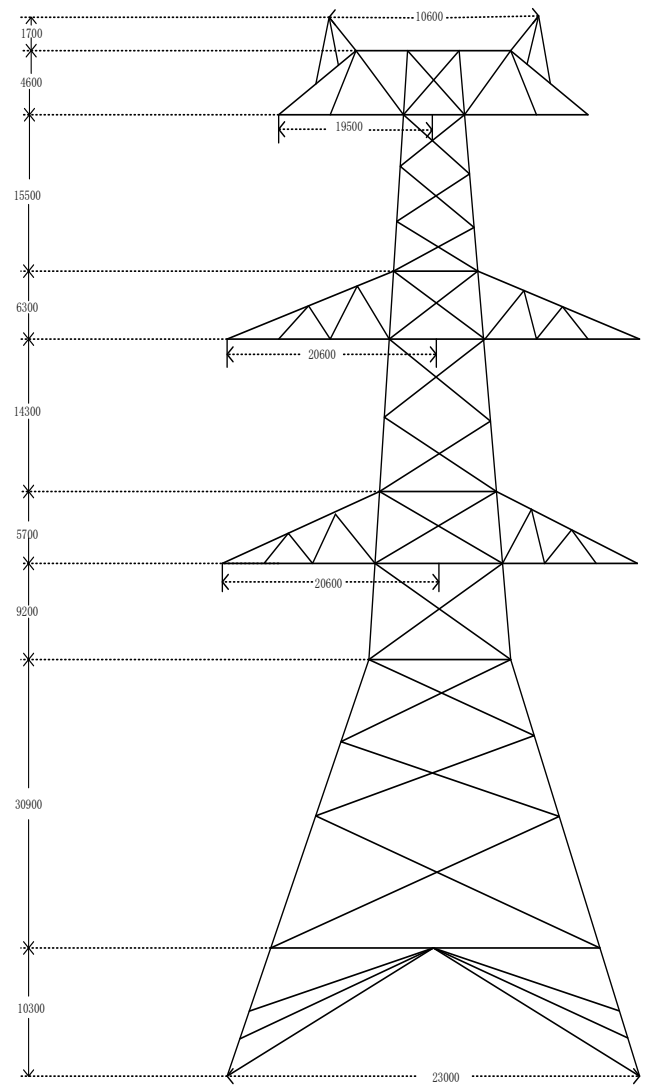

Fig.1 Outline dimension of tower type

\section{Comparison of calculation results of electric field strength}

\section{1. principle basis}

In the paper of "Research on Passive Interference of UHV AC Transmission Lines to Neighboring Stations", the mutual coupling effect between towers is discussed ${ }^{[8]}$. Since the mutual interference between the towers cannot ignored, here two towers are taken as an example. The electromagnetic interference coupling model as shown in Fig.4 is established. The two towers are respectively regarded as a transmitting antenna and a receiving antenna, and the output power of the transmitting antenna port is $\boldsymbol{P}_{t}$, the input power of the transmitting antenna port is $\boldsymbol{P}_{r}$.

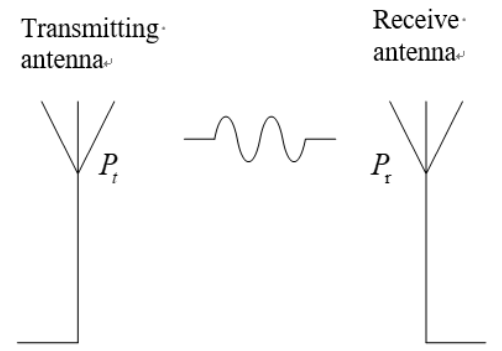

Fig.2 Radiative interference coupling model between two antennas

Define the coupling degree $\mathrm{c}$ between the far-field antenna as:

$$
c=\frac{\boldsymbol{P}_{r}}{\boldsymbol{P}_{t}}
$$

Fig. 3 is a schematic diagram showing the relative positions of the off-angles between the transmitting and receiving antennas. ${ }^{G_{r}}$ and ${ }^{G_{t}}$ :the gain of the receiving and transmitting antenna; $\theta_{1}$ and $\theta_{2}$ :the direction in which the radiation direction of the two antenna deviate from each other by the main beam of the opposite party; $\varphi_{1}$ and $\varphi_{2}$ :the angle between the main radiation direction of the two antennas and the horizontal plane; $\lambda$ :the working wavelength; $S$ :a polarization matching coefficient between the transmitting antenna and the receiving antenna; $L$ :the shortest distance between the two antennas.

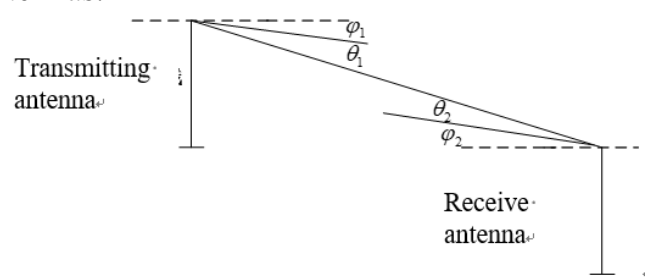

Fig.3 Mutual departure angle between transmitting and receiving antennas

So there are:

$$
P_{r}=\frac{\cos \theta_{1} \cos \theta_{2} P_{t} G_{t} G_{r} \lambda^{2} S}{(4 \pi L)^{2}}
$$

Finished up:

$$
c=\frac{\cos \theta_{1} \cos \theta_{2} G_{t} G_{r} \lambda^{2} S}{(4 \pi L)^{2}}
$$

Idealize all the parameters and organize them :

$$
c=\left(\frac{\lambda}{4 \pi d}\right)^{2}
$$

Equation (3) can be derived into the array, just need to $d$ rewritten into $d_{i j}$. It can be regarded as the interference coupling coefficient of the $\mathrm{i}$-th transmit 
antenna to the $\mathrm{j}$-th receive antenna, which has the following from:

$$
C=\left\{\begin{array}{l}
\left(\frac{\lambda}{4 \pi d_{11}}\right)^{2}\left(\frac{\lambda}{4 \pi d_{12}}\right)^{2} \ldots \ldots\left(\frac{\lambda}{4 \pi d_{1 n}}\right)^{2} \\
\left(\frac{\lambda}{4 \pi d_{21}}\right)^{2}\left(\frac{\lambda}{4 \pi d_{22}}\right)^{2} \ldots \ldots\left(\frac{\lambda}{4 \pi d_{2 n}}\right)^{2} \\
\left(\frac{\lambda}{4 \pi d_{n 1}}\right)^{2}\left(\frac{\lambda}{4 \pi d_{n 2}}\right)^{2} \ldots \ldots\left(\frac{\lambda}{4 \pi d_{n n}}\right)^{2}
\end{array}\right.
$$

The physical meaning of the elements on the diagonal is the degree of autotransformation of a single antenna element itself, and its own coupling to itself is 1 , so matrix $\mathrm{C}$ can be rewritten into

$$
C=\left\{\begin{array}{l}
1 \quad\left(\frac{\lambda}{4 \pi d_{12}}\right)^{2} \ldots \ldots\left(\frac{\lambda}{4 \pi d_{1 n}}\right)^{2} \\
\left(\frac{\lambda}{4 \pi d_{21}}\right)^{2}\left(\frac{\lambda}{4 \pi d_{22}}\right)^{2} \ldots \ldots\left(\frac{\lambda}{4 \pi d_{2 n}}\right)^{2} \\
\left(\frac{\lambda}{4 \pi d_{n 1}}\right)^{2}\left(\frac{\lambda}{4 \pi d_{n 2}}\right)^{2} \ldots \ldots \ldots 1
\end{array}\right.
$$

Taking into account the coupling interference coefficient between the arrays, the electric field strength of the i-th tower is multiplied by the mutual coupling interference coefficient with respect to the electromagnetic radiation field strength of the 0th tower to the far point. Vertically polarized plane wave incident angle is $\alpha . E_{i}$ is the Field strength for the $\mathrm{i}$-th tower at the required far-field point; $r_{0}, r_{i}$ the distance between the 0 th and $\mathrm{i}$-th towers to the required far field point

$$
\begin{gathered}
E_{i}=\left|E_{0}\right| \bullet \frac{r_{0}}{r_{i}} \boldsymbol{e}^{j\left(\frac{2 \pi\left(r_{i}-r_{0}\right)}{\lambda}-\frac{2 \pi i * d \sin \alpha}{\lambda}\right)} \\
=\left|E_{0}\right| \bullet \frac{r_{0}}{r_{i}} \boldsymbol{e}^{j \frac{2 \pi\left(r_{i}-r_{0}-i * d \sin \alpha\right)}{\lambda}} \\
E_{\text {all }}=E_{i} \bullet C
\end{gathered}
$$

The method of using the electromagnetic field strength of a single tower as a reference and multiplying the array influence coefficient to the obtain the far-field characteristics is accurate and effective from the perspective of simulation calculation. This method can greatly improve the calculation efficiency.

\subsection{Relationship between the protection distance and the height of the tower}

In order to ensure that the transmission line does not affect the communication quality of the radio station, this section analyzed the minimum distance between the two (ie protection distance, at this time $S E \leq 0.5$ ).

Figure 4 shows the variation of electric field strength with horizontal distance when the tower is at different heights. Point $\mathrm{A}$ is the maximum protection distance when the transmission line is at a certain span ( $S E \leq 0.5$ ), Figure 5 is the protective distance changes with the to tower.

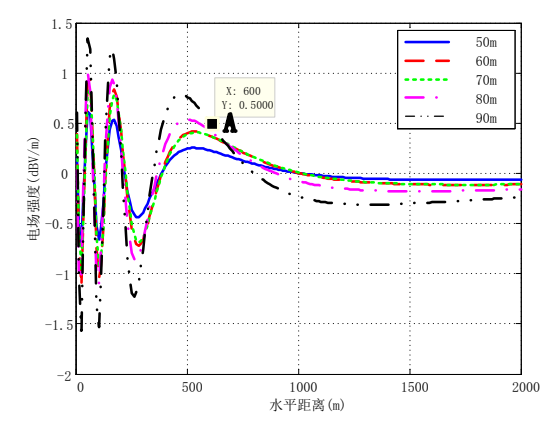

Fig.4 The electric field intensity level changes with horizontal distance

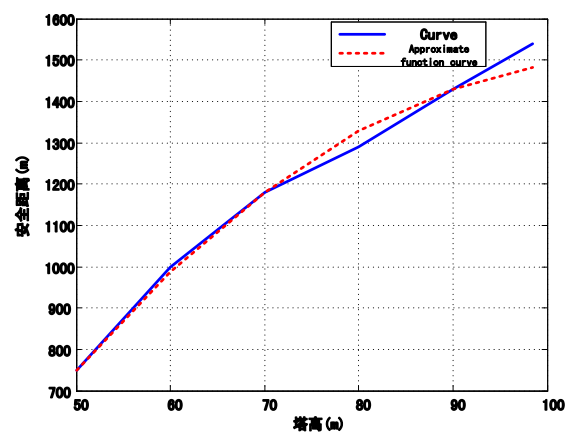

Fig.5 The protective distance changes with the span

In order to ensure that the protection distance is not affected by the height of the tower, this section uses the maximum protection distance. It can be seen from figure 4 that the higher the tower, the greater the protection distance required for the transmission line and the radio station. It can be seen from figure 5 that the relationship between the protection distance and the height of the tower in the error (error $\leqslant 5 \%$ ) range is as follows:

$$
S=-\frac{9}{40} h^{2}+\frac{97}{2} h-\frac{2225}{2} \quad(h>0 m)
$$

$S$ : protection distance between transmission lines and radio stations(ie $\boldsymbol{E} \leq 0.5 \mathrm{dBV} / \mathrm{m}$ ); $\boldsymbol{h}$ :tower height 。

In the actual project, if the incident frequency band of the plane wave is the short-wave frequency band, and the remaining factors are unchanged, the above formula can be used to derive the guard spacing at different tower heights. And regardless of the incident frequency of the plane wave, the protection distance curve will show a similar change law.

\section{Conclusion}

In this paper, the electric field strength calculation is carried out by applying the moment method under normal conditions. At the same time, the method of calculating the electromagnetic field of the transmission line is expounded. The electric field strength of the transmission line with different heights at the same working frequency and space at the same plane is calculated. The influence of tower heights on passive interference is different. Applying the approximation formula to summarizing the relationship between the tower height and protective distance.

(1)The height of the tower is an important factor affecting the protection distance (ie $S E \leq 0.5$ ) of the transmission line and the radio station, but weaker than 
the span. Under the same span, the distance between the transmission line and the radio station (ie $S E \leq 0.5$ ) depends on the height of the tower. The higher the height of the tower, the greater the required protection distance. The tower height and the protection distance satisfy the following fitting curve formula

$$
S=-\frac{9}{40} h^{2}+\frac{97}{2} h-\frac{2225}{2} \quad(h>0 m)
$$

(2)In practical engineering, according to the tower height and the protection distance formula, the avoidance distance of the transmission line and the radio station can be more accurately judged. It greatly reduces the time required for modeling and improves the accuracy of simulation calculations.

\section{Acknowledgement}

This work was supported by the Project of SGCC (GY71-16-011)

\section{References}

1. Yin hui, Zhang xiaoming, Wang yantao, et al.Research on 3D visualization of electromagnetic interference for Ultra-high Voltage transmission lines. High Voltage Engineering.40,12 (2014)

2. Tang bo, Yang jiawei Huang li, et al. Guard spacing of the air surveillance radar station to Ultrahigh Voltage transmission line.40.4(2018)

3. Tang bo, Sun rui, Zhao xiaoming, et al. Passive interference solution for UHV transmission line in very high frequency ban.Electric Power,49,10(2016)
4. Hill P C.Measurement of reradiation from lattice masts at V.H,F.[J].Proc Inst Elec Eng,111,12(1964)

5. Belroses J S,Lavrench W,Dunn J G,et al.The effects of reradiation from high rise buildings and transmission lines upon the radiation pattern of $\mathrm{MF}$ broadcasing antenna arrays[C]/Proceedings of AGARD/EPP Meeting .Spatind,Norway:[s.n.],1979.

6. Kamal Sarabandi,Moonsooo Park.A radar crosssection model for power lines at millmeter-wave frequency[J].IEEE Transactions on Antenna and Propagation,51,9(2003)

7. Truemen C W, Kubina S J. Scatting from power lines with the skywire insulated from the towers[J].IEEE Transactions on Broadcasting,40,2(1994)

8. Kinase. Theoretical analysis of reradiation characteristics of radio wave caused by transmission lines[ J] .NHK Technology Research, 20,3(1968)

9. Takeshita K,Takeshita S,Hashimoto H.Scattering characteristics of VHF/UHF television broadcasting waves by overhead oower transmission conductors[J]..IEEE Transactions on Electromagnetic Compatibility,21,1(1979)

10. Chen bin, Tang bo, Cao hongying, et al. Association Analysis of Passive Interference Resonance Impact Factors for Transmission Lines at Short-Wave Frequency,36,6(2015

11. Gan zheyuna, Wang yanhai, Zhang jiangong, et al, Suppression of $\mathrm{HF}$ passive interference in transmission lines by using magnetic tube . High Voltage Engineering, 43,5(2017) 